\title{
Factors That Cause and Affect Cancer Metastases, the Targeted Therapy and Potential Treatments
}

\author{
Yiming Liao*
}

\author{
Beijing SMIC Private High School, Beijing, China, 100163 \\ *Corresponding author.Email: yimingliao927@outlook.com
}

\begin{abstract}
The metastases are a series of mechanisms for circulating malignant tumor cells to travel and occupy distant organs. This includes infiltrating distant tissue, evading immune defenses, adapting to supportive niches, surviving as latent tumor-initiating seeds, and eventually breaking out to replace the host tissue[1]. Metastases like bone metastases can rapidly decline the survival quality and strongly affect the treatment protocol of the cancer patients. The original treatment will be unapplicable when metastases occur, therefore researching and gaining a better understanding of metastatic cancers' mechanisms is essential to cure or avoid cancer patients to endure tumor cell metastases. This review summarizes and develops based on the existing theories and hypotheses relevant to the intrinsic modification of cancer metastases. The promoting factors in the aggressive metastases had been characterized in the review aiming to provide a clear vision of potential and valuable research orientation regarding cancer metastases. Targeted therapy, immunotherapy, and potential treatment according to current studies will be summarized and depict. The paper finds that the knowledge of the process of cancer cell migration is now largely developed and several basic steps, such as invasion, survival, and colonization, have been established. A multifaceted and intact understanding of tumor cells and their subtle mechanism is expected in the following years.
\end{abstract}

Keywords: Cancer metastases, Targeted Therapy, Malignant tumor, Circulating tumor cells, Biomechanics.

\section{INTRODUCTION}

In clinical diagnosis, the classification for the last stage of cancer is described as nodal involvement, metastasis, and the size is greater than five centimeters while the stage before it does not involve metastasis. Through this classification, it is not hard to see that metastasis is a vital determinant of the severity of cancer. For primary cancer, surgery and chemotherapy can be used to block or even eradicate the tumor cells in the body. However, once the cancer cells start to metastasize, it is very difficult to track down each cancerous tissue and kill the cancer cells precisely with the existing medical methods. There are countless studies today on topics such as targeted therapies, but relatively minimal research on cancer prevention and diagnosis. More research went on how to suppress cancer cells that have already migrated, the author believes that solutions for early diagnosis and care of cancer patients are as important as the former. There are some research-based topics, such as how to screen cancer patients more effectively by combining clinical experience with the structure and function of the body. Is there a potentially more obvious response and basis for cancer determination in the body before cancer cells migrate? How can cancer cell metastisize be more effectively inhibited or even blocked when caring for patients with early stage cancer or those who have just undergone surgery? The statistics show that cancer metastasis is the number one contributor to death in cancer patients[2]. Therefore, researching and understanding cancer cell metastasis and the mechanisms behind it are essential for metastatic-preventive care and to develop more effective targeted therapeutic regimens and drugs. Thus, this paper summarizes and develops based on the existing theories and hypotheses relevant to the intrinsic modification of cancer metastases. The promoting factors in the aggressive metastases had been characterized in the review aiming to provide a clear vision of potential and valuable research orientation regarding cancer metastases. Targeted therapy, immunotherapy, and potential treatment according to current studies will be summarized and depict. This research direction could provide more effective treatment and care for future cancer patients and reduce the risk of death from cancer. 


\section{METASTASIS'S MECHANISMS}

Metastasis is a set of sequential, discrete, and complex biological procedures that leads to the colonization and formation of secondary tumors in distant organs from the primary tumor. This process can be roughly divided into three parts: invasion to the bloodstream or lymphatic channel, survival in the circulation, and colonizing into tissues and to initiate a new tumor.[3]

\subsection{Invasion}

Invasion refers to the process when tumor cells break off from the primitive site of cancer and enter the basement membrane of blood or lymph vessels. Cancer cell secretes enzymes name matrix metalloproteinase (MMP), and this substance helps tumor cells to degrade the collagenous extracellular matrix (ECM). When a tumor cell is broken off from the main tumor, then MMPs will enter the extracellular space. The migrating cell will be surrounded by MMPs and move toward bloodstreams and lymphatic channels. MMPs play a major role in creating and opening access to epithelial cells by their enzymatic action, which allows the tumor cells to enter the vessels through the tight junctions of the epithelial cells.

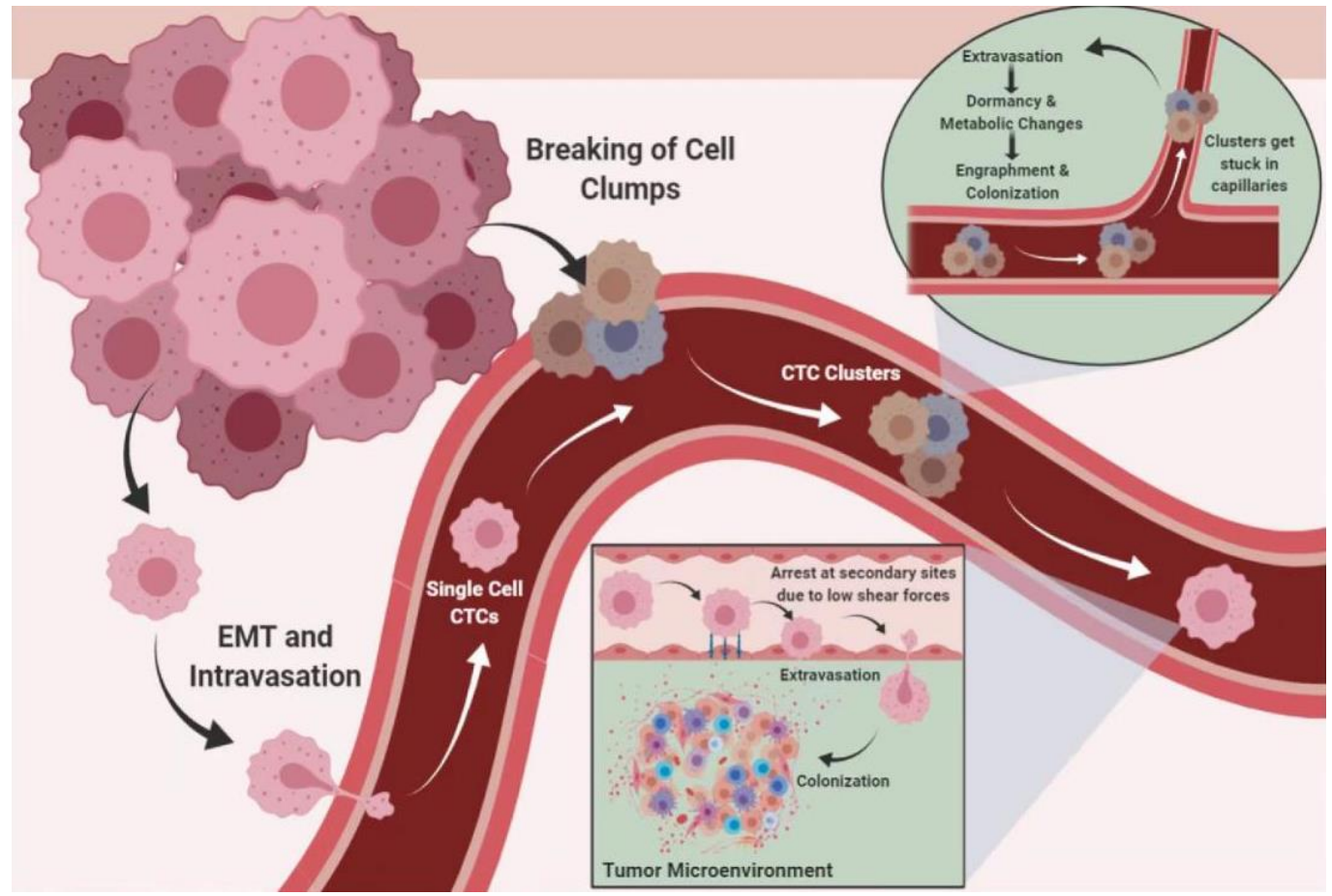

Figure 1 Cancer cells circulate as single units or in clusters. [8]

\subsection{Survival in the Circulation}

After entering the bloodstreams or the lymphatic channel, the circulating tumor cells (CTCs) will become clusters and bind with platelets to protect themselves.
CTCs' ability to endure oxidative stress is by exposing themselves to oxidative stress, the body' $\mathrm{s}$ immune system, and the sheer force in the circulatory patterns. Coating themselves with platelets is the common strategy for CTCs to survive in the blood circulation.[1]

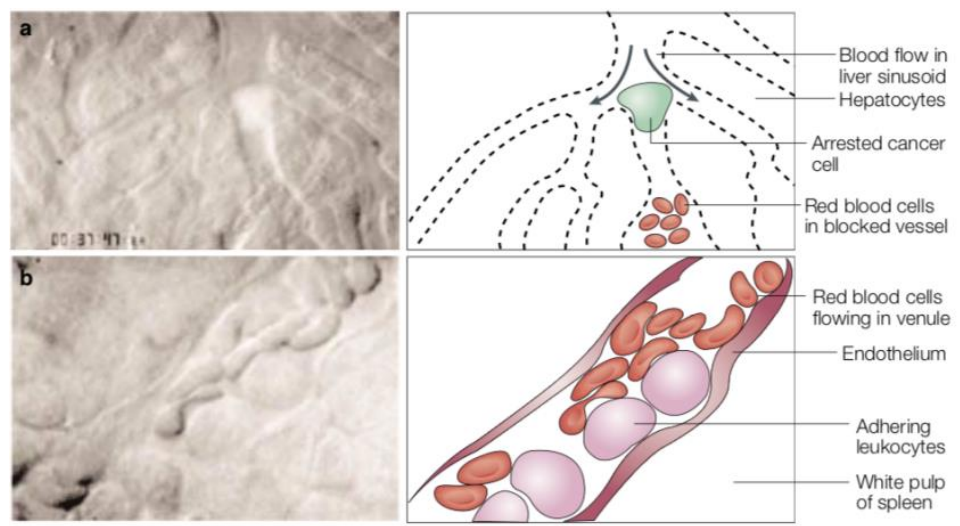

Figure2. Cancer and normal cells arrest in circulation [9] 


\subsection{Colonization}

Before entering the new tissue and initiating a new tumor, CTCs will be ensnared by capillaries. Due to the mechanisms of venous circulation, CTCs tend to be trapped in the lung and liver, and contribute to the high rate of metastasis and forming a secondary tumor in these organs. Nevertheless, some CTCs will still pass the retention of these filters and enter other organs by arterial circulation. Once CTCs make secure attachments with the epithelial lining outside the targeted tissue, they will exit the vessel and enter the new site. These CTCs will leave an open pathway for following tumor cells to enter the metastasis site. CTCs can also start growing in the vessel and resulting in embolus growth that could rupture the vessels and extravasate by breaching vascular walls to achieve their colonization.[1]

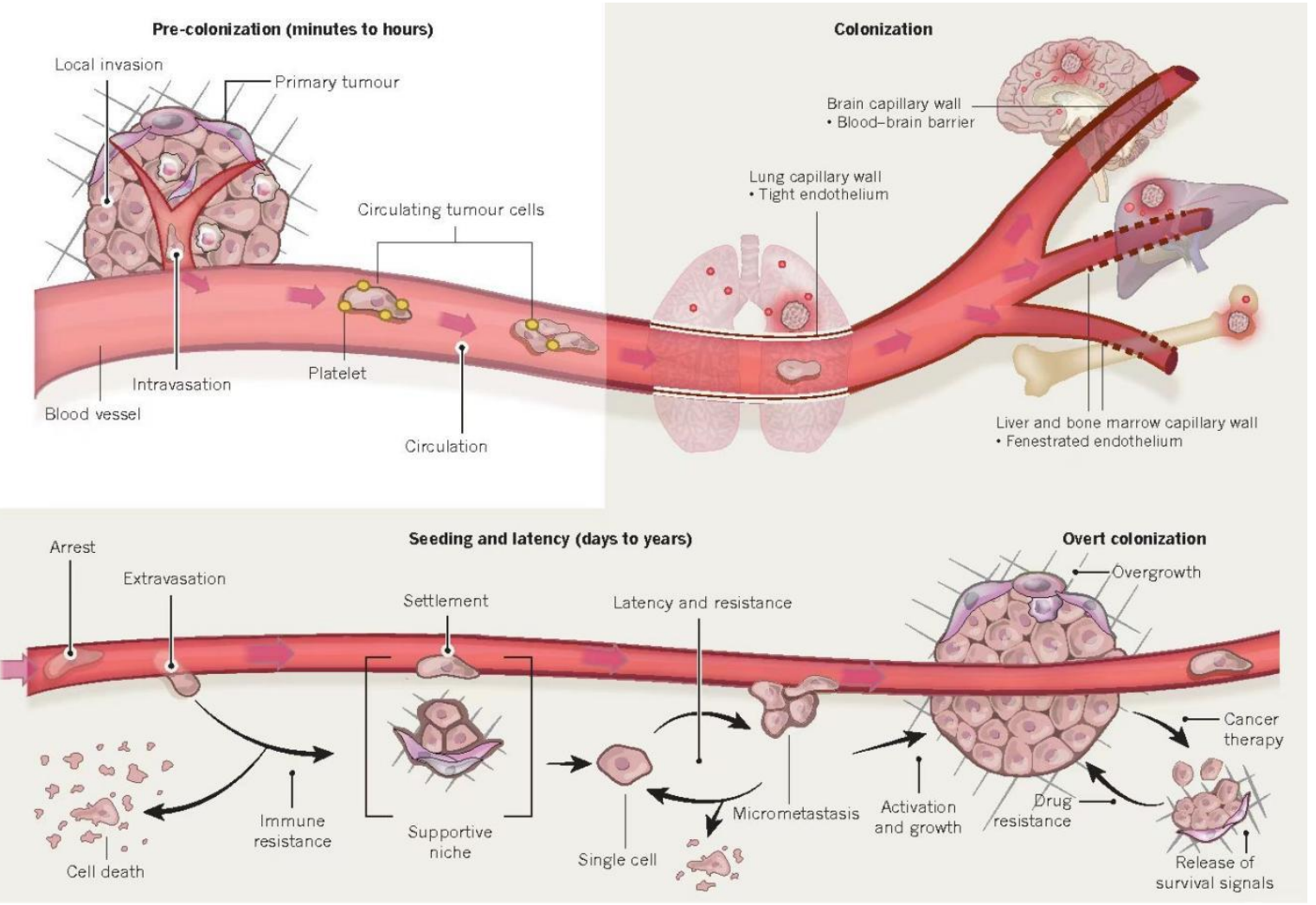

Figure 3 Metastatic colonization [1]

\section{OSMOTIC PRESSURE AND CANCER THERAPY}

Researchers had made hypotheses that osmotic pressure is the driving force for continuous cell division. The mutations of cancer genes or inactivation of oncogenes can directly or indirectly promote tumor cell proliferation. It regulates the rate of change in intracellular permeability tumor cell proliferation by regulating the rate of change in intracellular permeability. Researchers gave evidence to support the hypothesis in the paper, but no specific experimental evidence had been provided to demonstrate that the hypothesis is dependable. Assuming that this hypothesis is practical, then the method of regulating intracellular osmolarity can be applied to the treatment of malignant tumors. The possibility of the suppression of mutated or amplified oncogenes turns to reduce the rate of increase in tumor cell osmolality, thereby inhibiting the growth of tumor cells exists. Clinical studies can take advantage of the vulnerability of tumor cells to rupture when their osmotic pressure increases rapidly by further increasing the osmotic pressure within the tumor cells to cause them to rupture and die. This is a very promising study for the development of broad-spectrum therapeutic agents for malignant tumors.[4]

\section{TARGETED THERAPY}

The term "targeted therapy" refers to the drugs of cancer treatment that interfere with the specific molecule that plays an essential role in controlling the growth and apoptosis signals in tumor cells. The study shows that these molecules are typically kinase proteins.[5]

The typically targeted therapies are small molecule protein TKIs, MoAbs, and vaccines.[6] Small molecules are called small molecules because they could enter the plasma membrane, and examples of small molecule drugs are pazopanib, erlotinib, imatinib, and sunitinib. When they are practical used in clinical cases, it requires consideration of food and drug interactions since small molecule drugs are orally taken. Monoclonal antibodies 
work outside the cell, blocking the interaction between signaling molecules and receptors. MoAbs can also release radioactive molecules or cytotoxins into cancer cells and cause cell death. For vaccines, instead of targeting cancer cells specifically, they broadly activate the body's immune system to recognize and attack tumor cells.

\section{PREVENTION OF METASTASIS}

Despite the discussion about the promising aspects of clinical therapies about metastasized cancer this paper had just covered, the intensification the preventing metastasis is a more critical problem to solve. Many other researchers also recognized that compare to the study of therapy, the prevention strategies in cancer metastasis are rarely discussed in scientific publications (less than 4\%).[7] The clinical and theoretical research orientating the topic of treating metastasized cancer focus on themes about restraining the cancer growth, the methods to reduce tumors size, and targeted therapy with their receptors. Since the most hazardous event that causes cancer patients, death is metastases, and numerous successful treatment cases of metastasized cancer all rely on early detection and metastaticpreventive care. This phenomenon demonstrates that health care and restriction on patients and their tumor cells might be a more effective and more worthwhile research direction of cancer treatments.

\section{CONCLUSION}

This paper examines the effects and mechanisms of cancer migration on patients, gives a basic overview of current cancer treatment methods and trends, analyzes the developable directions in the field of cancer research, and presents the significance of current relatively unprevailing research topics, which serve to provide research directions for related subjects. It can be concluded that the knowledge of the process of cancer cell migration is now largely developed and several basic steps, such as invasion, survival, and colonization, have been established. Research based on this process has a great potential to be useful for finding therapeutic targets. At the same time, the causes of cancer cell migration are also significant for cancer therapy. Because the current research findings on the underlying causes of cancer cell migration are not abundant and essential enough. Some scientists have proposed the hypothesis that osmotic pressure is the driving force of cancer cell division, for which the hypothesis extends many promising therapeutic modalities, and more attention and experiments need to be paid.

\section{ACKNOWLEDGMENTS}

I would like to express my gratitude to all those who helped me with this paper and gave me the opportunity to research this topic and write this paper. In particular, I would like to thank Dr. Cheng for helping me research the basics of this thesis and deepening my interest and passion in the field of molecular biology and life sciences. I am also very grateful to my thesis advisor, Ms. Han, for contributing many constructive suggestions to my thesis and providing me with encouragement and supervision.

\section{REFERENCES}

[1] Massagué, J., \& Obenauf, A. C.. Metastatic Colonization By Circulating Tumour Cells. Nature, 529(7586), 2016:298-306.

[2] Siegel, R. L., Miller, K. D., \& Jemal, A.. Cancer statistics. CA: A Cancer Journal for Clinicians, 2020.

[3] Steeg, P. S.. Tumor metastasis: mechanistic insights and clinical challenges. Nature Medicine, 12(8), 2006: 895-904.

[4] ZHANG Feng,JIANG Runmin,WANG He,et al.Uncontyollable of osmotic pressure may cause cancer[J].,2020,(03):63-67.

[5] Sawyers, C.. Targeted cancer therapy. Nature, 432(7015), 2004: 294-297.

[6] Wujcik, D.. Science and Mechanism of Action of Targeted Therapies in Cancer Treatment. Seminars in Oncology Nursing, 30(3), 2014:139-146.

[7] Meirson, T., Gil-Henn, H., \& Samson, A. O.. Invasion and metastasis: the elusive hallmark of cancer. Oncogene, 2019

[8] Fares, J., Fares, M. Y., Khachfe, H. H., Salhab, H. A., \& Fares, Y.. Molecular principles of metastasis: a hallmark of cancer revisited. Signal Transduction and Targeted Therapy, 5(1), 2020

[9] Chambers, A. F., Groom, A. C. \& MacDonald, I. C. Dissemination and growth of cancer cells in metastatic sites. Nature Rev. Cancer 2, 563-572, 2002. 